\title{
Approximate Kelvin-Voigt Fluid Driven by an External Force Depending on Velocity with Distributed Delay
}

\author{
Yantao Guo, ${ }^{1,2}$ Shuilin Cheng, ${ }^{3}$ and Yanbin Tang ${ }^{1}$ \\ ${ }^{1}$ School of Mathematics and Statistics, Huazhong University of Science and Technology, Wuhan, Hubei 430074, China \\ ${ }^{2}$ School of Mathematics and Statistics, Xuchang University, Xuchang, Henan 461000, China \\ ${ }^{3}$ School of Statistics and Mathematics, Zhongnan University of Economics and Law, Wuhan, Hubei 430073, China
}

Correspondence should be addressed to Yanbin Tang; tangybhust@sina.com

Received 23 March 2015; Accepted 2 May 2015

Academic Editor: Luca Gori

Copyright (C) 2015 Yantao Guo et al. This is an open access article distributed under the Creative Commons Attribution License, which permits unrestricted use, distribution, and reproduction in any medium, provided the original work is properly cited.

We consider the approximate 3D Kelvin-Voigt fluid driven by an external force depending on velocity with distributed delay. We investigate the long time behavior of solutions to Navier-Stokes-Voigt equation with a distributed delay external force depending on the velocity of fluid on a bounded domain. By a prior estimate and a contractive function, we give a sufficient condition for the existence of pullback attractor of NSV equation.

\section{Introduction}

In this paper, we consider 3D Navier-Stokes-Voigt (NSV) equation with a distributed delay external force depending on the velocity of the fluid:

$$
\begin{aligned}
& u_{t}-\alpha^{2} \Delta u_{t}-v \Delta u+(u \cdot \nabla) u+\nabla p \\
& =\int_{-h}^{0} G(s, u(t+s)) d s, \\
& \operatorname{div} u(t, x)=0, \quad(t, x) \in[\tau,+\infty) \times \Omega, \\
& u(t, x)=0, \quad(t, x) \in[\tau,+\infty) \times \partial \Omega, \\
& u(t, x)=\phi(t-\tau, x), \quad t \in[\tau-h, \tau], \quad x \in \Omega,
\end{aligned}
$$

where $u=\left(u_{1}, u_{2}, u_{3}\right)$ is the velocity field of the fluid, $p$ is the pressure, $v>0$ is the kinematic viscosity, $\alpha>0$ is the length scale parameter of the elasticity of the fluid, the external force $G$ and initial velocity field $\phi$ are defined in the interval of time $[-h, 0]$, where $h$ is a fixed positive number and $\Omega$ is a bounded smooth domain of $R^{3}$.

The NSV equation was introduced by Oskolkov [1] to give an approximate description of the Kelvin-Voigt fluid and was proposed as a regularization of 3D Navier-Stokes equation for the purpose of direct numerical simulations in
[2]. Since the term $-\alpha^{2} \Delta u_{t}$ changes the parabolic character of the equation, the NSV equation being well posed in 3D, many authors have studied the long time dynamics of this model. Kalantarov and Titi [3] investigated the existence of the global attractor, the estimation of the upper bounds for the number of determining modes, and the dimension of global attractor of the semigroup generated by the equations. By a useful decomposition method, Yue and Zhong [4] proved the asymptotic regularity of solution of NSV equation and obtained the existence of the uniform attractor; they also described the structure of the uniform attractor and its regularity. García-Luengo et al. [5] investigated the existence and relationship between minimal pullback attractor for the universe of fixed bounded sets and universe given by a tempered condition.

Partial differential equations with delays arise from various fields, like physics, control theory, and so on (see, e.g., [6-10]); the unknown functions depend on not only present stage but also some past stage. The existence and stability of solution and global attractor for Navier-Stokes equation with discrete delay were established in [11-13]. The existence of pullback attractors in $C_{H_{0}^{1}}$ and $C_{H_{0}^{1} \cap H^{2}}$ was proved for the processes associated with nonclassical diffusion equations with variable bounded delay in $[14,15]$. Delay effect has been considered on an unbounded domain in [16]. The existence of 
pullback attractor for a Navier-Stokes equation with infinite discrete delay effect was studied in [17].

The aim of this paper is to investigate the NSV equation with a distributed delay, instead of the discussions with finite delays in the references. Our purpose is twofold. We first show the existence and uniqueness of solution to NSV equation (1) with a distributed delay; then we prove the existence of pullback attractor for the process generated by the NSV equation (1).

This paper is organized as follows. In Section 2, we give some preliminary results and prove existence of solution to NSV equation with a distributed delay. In Section 3, we derive the existence of pullback attractor by prior estimates and contractive functions.

\section{Existence of Solutions}

In order to prove the existence of solutions to problem (1), we define the function spaces

$$
\mathscr{V}=\left\{u \in\left(C_{0}^{\infty}(\Omega)\right)^{3}, \operatorname{div} u=0\right\}
$$

$H$ is the closure of $\mathscr{V}$ in $\left(L^{2}(\Omega)\right)^{3}$ with the inner product $(\cdot, \cdot)$ and associate norm $|\cdot|, V$ is the closure of $\mathscr{V}$ in $\left(H_{0}^{1}(\Omega)\right)^{3}$ with scalar product $((\cdot, \cdot))$ and associate norm $\|\cdot\|$, where

$$
((u, v))=\sum_{i, j=1}^{3} \int_{\Omega} \frac{\partial u_{j}}{\partial x_{i}} \frac{\partial v_{j}}{\partial x_{i}} d x, \quad \forall u, v \in\left(H_{0}^{1}(\Omega)\right)^{3}
$$

it follows that $V \subset H \equiv H^{\prime} \subset V^{\prime}$, where the injections are dense and compact. We will use $\|\cdot\|_{*}$ for the norm in $V^{\prime}$ and $\langle\cdot, \cdot\rangle$ for the duality pairing between $V$ and $V^{\prime}$.

Define the linear continuous operator $A: V \rightarrow V^{\prime}$ as

$$
\langle A u, v\rangle=((u, v)), \quad \forall u, v \in V .
$$

We denote $D(A)=\{u \in V, A u \in H\}$; one has that $D(A)=$ $\left(H^{2}(\Omega)\right)^{3} \cap V$ and $A u=-P \Delta u$, for all $u \in D(A)$ is the Stokes operator, where $P$ is the orthoprojector from $\left(L^{2}(\Omega)\right)^{3}$ onto $H$; also denote $C_{H}=C^{0}([-h, 0] ; H)$ and $C_{V}=C^{0}([-h, 0] ; V)$.

Define the trilinear form $b$ on $V \times V \times V$ by

$$
b(u, v, w)=\sum_{i, j=1}^{3} \int_{\Omega} u_{i} \frac{\partial v_{j}}{\partial x_{i}} w_{j} d x, \quad \forall u, v, w \in V
$$

and the operator $B: V \times V \rightarrow V^{\prime}$ as

$$
\langle B(u, v), w\rangle=b(u, v, w), \quad \forall u, v, w \in V,
$$

and denote $B(u)=B(u, u)$.

The trilinear form $b$ satisfies that

$$
\begin{aligned}
& b(u, v, w)=-b(u, w, v), \\
& b(u, v, v)=0
\end{aligned}
$$

We also recall that there exists a constant $C$ depending only on $\Omega$ such that

$$
\begin{aligned}
|b(u, v, w)| \leq C\|u\|^{1 / 2}|A u|^{1 / 2}\|v\||w|, & \\
u \in D(A), & v \in V, w \in H, \\
|b(u, v, w)| \leq C\|u\|\|v\||w|^{1 / 2}\|w\|^{1 / 2}, & \\
u & \in V, \quad v \in V, w \in V, \\
|b(u, v, w)| \leq C|u|^{1 / 2}\|u\|^{1 / 2}\|v\|\|w\|, & \\
u & \in V, \quad v \in V, w \in V .
\end{aligned}
$$

For the term containing the time delay, $G: R \times H \rightarrow H$ satisfies that

$$
\begin{aligned}
& \left(H_{1}\right) G(\cdot, u): R \rightarrow H \text { is a measurable function, } \\
& \left(H_{2}\right) G(t, 0)=0 \text { for all } t \in R
\end{aligned}
$$

$\left(H_{3}\right)$ there exists a positive constant $L$, such that $\forall R>$ 0 ; if $|u|<R$ and $|v|<R$, then

$$
|G(t, u)-G(t, v)|^{2} \leq L|u-v|^{2}
$$

Remark 1. Hypotheses $\left(H_{2}\right)-\left(H_{3}\right)$ imply that $|G(t, u)|^{2} \leq$ $L|u|^{2}$, so we have $|G(t, u)|^{2} \in L^{\infty}(\tau, T)$ for $|u|<R$.

Problem (1) can be rewritten as

$$
\begin{aligned}
& \frac{\partial}{\partial t}\left(u+\alpha^{2} A u\right)+v A u+B(u, u) \\
& \quad=\int_{-h}^{0} G(s, u(t+s)) d s, \\
& u(t, x)=\phi(t-\tau, x), \quad t \in[\tau-h, \tau], x \in \Omega ;
\end{aligned}
$$

then we get the existence of solution to problem (10).

Theorem 2. Let $\phi \in C_{V}$, let $G: R \times H \rightarrow H$ satisfy the hypotheses $\left(H_{1}\right)-\left(H_{3}\right)$, and let $\tau \in \mathrm{R}$. Then, $\forall T>\tau$; there exists a unique weak solution to (10) such that

$$
\begin{gathered}
u(t, x) \in C([\tau-h, T] ; V), \\
\frac{\partial u(t, x)}{\partial t} \in L^{2}(\tau, T ; V) .
\end{gathered}
$$

Moreover, if $\phi \in C_{D(A)}$, then problem (10) admits a strong solution.

Proof. Consider the Galerkin approximations for problem (10):

$$
\begin{aligned}
& \frac{d}{d t}\left(u^{m}+\alpha^{2} A u^{m}\right)+v A u^{m}+B\left(u^{m}, u^{m}\right) \\
& \quad=\int_{-h}^{0} G\left(s, u^{m}(t+s)\right) d s,
\end{aligned}
$$


Discrete Dynamics in Nature and Society

3

where $u^{m}=\sum_{j=1}^{m} u_{j}^{m} e_{j}, A u^{m}=\sum_{j=1}^{m} \lambda_{j} u_{j}^{m} e_{j}$, and $e_{j}$ and $\lambda_{j}$ are the corresponding orthonormal eigenfunction and eigenvalues of operator $A$, respectively; then,

$$
\begin{aligned}
\left\|u^{m}\right\|^{2} & =\sum_{j=1}^{m} \lambda_{j}\left(u_{j}^{m}\right)^{2}, \\
\left|A u^{m}\right|^{2} & =\sum_{j=1}^{m} \lambda_{j}^{2}\left(u_{j}^{m}\right)^{2}, \\
\left|u^{m}\right|^{2} & =\sum_{j=1}^{m}\left(u_{j}^{m}\right)^{2} .
\end{aligned}
$$

We now derive a prior estimate for the Galerkin approxinmate solution. Multiplying (12) by $u_{j}^{m}$, summing from $j=1$ to $m$ and using the fact

$$
\left(B\left(u^{m}, u^{m}\right), u^{m}\right)=b\left(u^{m}, u^{m}, u^{m}\right)=0,
$$

we obtain that, for a.e. $t>\tau$,

$$
\begin{aligned}
& \frac{d}{d t}\left(\left|u^{m}\right|^{2}+\alpha^{2}\left\|u^{m}\right\|^{2}\right)+2 v\left\|u^{m}\right\|^{2} \\
& \quad=2\left(\int_{-h}^{0} G\left(s, u^{m}(t+s)\right) d s, u^{m}\right) \\
& \quad \leq\left|\int_{-h}^{0} G\left(s, u^{m}(t+s)\right) d s\right|^{2}+\left|u^{m}\right|^{2} .
\end{aligned}
$$

$$
\begin{gathered}
\left|u^{m}(t)\right|^{2}+\alpha^{2}\left\|u^{m}(t)\right\|^{2}+2 v \int_{\tau}^{t}\left\|u^{m}(s)\right\|^{2} d s \\
\leq\left|u^{m}(\tau)\right|^{2}+\alpha^{2}\left\|u^{m}(\tau)\right\|^{2} \\
+\int_{\tau}^{t}\left|\int_{-h}^{0} G\left(s, u^{m}(r+s)\right) d s\right|^{2} d r \\
+\int_{\tau}^{t}\left|u^{m}(r)\right|^{2} d r .
\end{gathered}
$$

Remark 1 implies that

$$
\begin{aligned}
& \int_{\tau}^{t}\left|\int_{-h}^{0} G\left(s, u^{m}(r+s)\right) d s\right|^{2} d r \\
& \quad \leq \int_{-h}^{0} \int_{\tau}^{t}\left|G\left(s, u^{m}(r+s)\right)\right|^{2} d r d s \\
& \quad \leq \int_{-h}^{0} \int_{\tau}^{t} L\left|u^{m}(r+s)\right|^{2} d r d s \\
& \quad \leq L \int_{-h}^{0} \int_{\tau+s}^{t+s}\left|u^{m}(r)\right|^{2} d r d s \\
& \quad \leq L \int_{-h}^{0}\left(\int_{\tau-h}^{\tau}\left|u^{m}(r)\right|^{2} d r+\int_{\tau}^{t}\left|u^{m}(r)\right|^{2} d r\right) d s \\
& \quad \leq L h^{2}\|\phi\|_{C_{H}}^{2}+L h \int_{\tau}^{t}\left|u^{m}(r)\right|^{2} d r .
\end{aligned}
$$

Then, $\forall t \in(\tau, T)$ and

$$
\begin{aligned}
& \left|u^{m}(t)\right|^{2}+\alpha^{2}\left\|u^{m}(t)\right\|^{2}+2 v \int_{\tau}^{t}\left\|u^{m}(s)\right\|^{2} d s \\
& \leq(L h+2) \int_{\tau}^{t}\left|u^{m}(s)\right|^{2} d s+L h^{2}\|\phi\|_{C_{H}}^{2}+|\phi|_{C_{H}}^{2} \\
& \quad+\alpha^{2}\|\phi\|_{C_{V}}^{2} .
\end{aligned}
$$

So, we have

$$
\begin{aligned}
\left|u^{m}(t)\right|^{2} \leq & C \int_{\tau}^{t}\left|u^{m}(s)\right|^{2} d s+C\|\phi\|_{C_{H}}^{2}+|\phi|_{C_{H}}^{2} \\
& +\alpha^{2}\|\phi\|_{C_{V}}^{2} .
\end{aligned}
$$

The Gronwall inequality implies that

$$
\left|u^{m}(t)\right|^{2} \leq C
$$

Putting (20) into the right-hand side of (18), we have

$$
\alpha^{2}\left\|u^{m}(t)\right\|^{2}+2 v \int_{\tau}^{t}\left\|u^{m}(s)\right\|^{2} d s \leq C, \quad \forall t \in(\tau, T) .
$$

This implies that

$$
\left\{u^{m}\right\} \text { is bounded in } L^{\infty}(\tau, T ; V) \cap L^{2}(\tau, T ; V) .
$$

Now, multiplying (12) by $\partial_{t} u^{m}$ and integrating over $\Omega$, we have

$$
\begin{aligned}
& \left|\partial_{t} u^{m}\right|^{2}+\alpha^{2}\left\|\partial_{t} u^{m}\right\|^{2}+\frac{v}{2} \frac{d}{d t}\left\|u^{m}\right\|^{2} \\
& \leq\left|b\left(u^{m}, u^{m}, \partial_{t} u^{m}\right)\right| \\
& \quad+\left|\left(\int_{-h}^{0} G\left(s, u^{m}(t+s)\right) d s, \partial_{t} u^{m}\right)\right| \\
& \leq c\left\|u^{m}\right\|^{3 / 2}\left|u^{m}\right|^{1 / 2}\left\|\partial_{t} u^{m}\right\| \\
& \quad+\frac{1}{2}\left|\int_{-h}^{0} G\left(s, u^{m}(t+s)\right) d s\right|^{2}+\frac{1}{2}\left|\partial_{t} u^{m}\right|^{2} ;
\end{aligned}
$$

since

$$
\left\|u^{m}\right\|^{3 / 2}\left|u^{m}\right|^{1 / 2}\left\|\partial_{t} u^{m}\right\| \leq c\left\|u^{m}\right\|^{3}\left|u^{m}\right|+\frac{\alpha^{2}}{2}\left\|\partial_{t} u^{m}\right\|^{2} ;
$$

then

$$
\begin{aligned}
& \left|\partial_{t} u^{m}\right|^{2}+\alpha^{2}\left\|\partial_{t} u^{m}\right\|^{2}+v \frac{d}{d t}\left\|u^{m}\right\|^{2} \\
& \quad \leq c\left\|u^{m}\right\|^{3}\left|u^{m}\right|+\left|\int_{-h}^{0} G\left(s, u^{m}(t+s)\right) d s\right|^{2},
\end{aligned}
$$


integrating the above inequality from $\tau$ to $t$, by (17), (20), and (22) we have

$$
\begin{gathered}
\int_{\tau}^{t}\left(\left|\partial_{t} u^{m}\right|^{2}+\alpha^{2}\left\|\partial_{t} u^{m}\right\|^{2}\right) d s+v\left\|u^{m}(t)\right\|^{2} \\
\leq v\left\|u^{m}(\tau)\right\|^{2}+c \int_{\tau}^{t}\left\|u^{m}\right\|^{3}\left|u^{m}\right| d s \\
\quad+\int_{\tau}^{t}\left|\int_{-h}^{0} G\left(s, u^{m}(r+s)\right) d s\right|^{2} d r \\
\leq v\|\phi(\tau)\|^{2}+c \int_{\tau}^{t}\left\|u^{m}\right\|^{3}\left|u^{m}\right| d s+c\|\phi\|_{C_{H}}^{2} \\
\quad+c \int_{\tau}^{t}\left|u^{m}(r)\right|^{2} d r \\
\leq v\|\phi\|_{C_{V}}^{2}+c \int_{\tau}^{t}\left\|u^{m}\right\|^{3}\left|u^{m}\right| d s+c\|\phi\|_{C_{H}}^{2} \\
\quad+c \int_{\tau}^{t}\left|u^{m}(r)\right|^{2} d r .
\end{gathered}
$$

Since $\left\{u^{m}\right\}$ is bounded in $L^{\infty}(\tau, T ; V) \cap L^{2}(\tau, T ; V)$, we obtain that

$$
\left\{\partial_{t} u^{m}\right\} \text { is bounded in } L^{2}(\tau, T ; V) .
$$

By the Faedo-Galerkin scheme, for example, see $[14,18]$, according to the estimates (22) and (27), we can get existence of the weak solution; here we omit the details.

We next consider the uniqueness of solution. Let $u, v$ be two solutions to problem (10) corresponding the initial data $\phi$ and $\psi$, respectively.

Denote $w=u-v$; then, we have

$$
\begin{aligned}
\frac{\partial}{\partial t} & \left(w+\alpha^{2} A w\right)+v A w+B(u, u)-B(v, v) \\
& =\int_{-h}^{0} G(s, u(t+s)) d s-\int_{-h}^{0} G(s, v(t+s)) d s .
\end{aligned}
$$

Multiplying (28) by $w$ and integrating over $\Omega$, we obtain

$$
\begin{aligned}
\frac{1}{2} \frac{d}{d t} & \left(|w|^{2}+\alpha^{2}\|w\|^{2}\right)+v\|w\|^{2}+(B(u, u), w) \\
& -(B(v, v), w) \\
= & \left(\int_{-h}^{0} G(s, u(t+s)) d s-\int_{-h}^{0} G(s, v(t+s)) d s, w\right) .
\end{aligned}
$$

Notice that

$$
\begin{aligned}
&(B(u, u), w)-(B(v, v), w) \\
&= b(u, u, u-v)-b(v, u, u-v)+b(v, u, u-v) \\
&-b(v, v, u-v) \\
&= b(u-v, u, u-v)-b(v, u-v, u-v) \\
&= b(w, u, w) .
\end{aligned}
$$

Substituting (30) into (29) and integrating from $\tau$ to $t$, we get

$$
\begin{aligned}
& |w(t)|^{2}+\alpha^{2}\|w(t)\|^{2}+2 v \int_{\tau}^{t}\|w(s)\|^{2} d s-|w(\tau)|^{2} \\
& -\alpha^{2}\|w(\tau)\|^{2} \leq \int_{\tau}^{t}|b(w, u, w)| d s+\int_{\tau}^{t}|w(s)|^{2} d s \\
& \quad+\int_{\tau}^{t} \mid \int_{-h}^{0} G(s, u(r+s)) d s \\
& -\left.\int_{-h}^{0} G(s, v(r+s)) d s\right|^{2} d r .
\end{aligned}
$$

$\left(H_{3}\right)$ implies that

$$
\begin{aligned}
& \int_{\tau}^{t}\left|\int_{-h}^{0} G(s, u(r+s)) d s-\int_{-h}^{0} G(s, v(r+s)) d s\right|^{2} d r \\
& \quad \leq \int_{-h}^{0} \int_{\tau}^{t}|G(s, u(r+s))-G(s, v(r+s))|^{2} d r d s \\
& \quad \leq \int_{-h}^{0} \int_{\tau}^{t}|G(s, u(r+s))-G(s, v(r+s))|^{2} d r d s \\
& \quad \leq L \int_{-h}^{0} \int_{\tau}^{t}|u(r+s)-v(r+s)|^{2} d r d s \\
& \quad \leq L \int_{-h}^{0} \int_{\tau+s}^{t+s}|u(r)-v(r)|^{2} d r d s \\
& \quad \leq L \int_{-h}^{0}\left(\int_{\tau-h}^{\tau}|u(r)-v(r)|^{2} d r\right. \\
& \left.\quad+\int_{\tau}^{t}|u(r)-v(r)|^{2} d r\right) d s \leq L h^{2}\|\phi-\psi\|_{C_{H}}^{2} \\
& \quad+L h \int_{\tau}^{t}|w(s)|^{2} d s .
\end{aligned}
$$

As the property of operator $b$ and Poincaré, we have

$$
\begin{aligned}
\int_{\tau}^{t}|b(w, u, w)| d s & \leq C \int_{\tau}^{t}\|w\|^{3 / 2}|w|^{1 / 2}\|u\| d s \\
& \leq C \int_{\tau}^{t}\|w\|^{2}\|u\| d s
\end{aligned}
$$


Substituting (32) and (33) into (31), we get

$$
\begin{gathered}
|w(t)|^{2}+\alpha^{2}\|w(t)\|^{2}+2 v \int_{\tau}^{t}\|w(s)\|^{2} d s \\
\leq \int_{\tau}^{t}\|w\|^{2}\|u\| d s+L h^{2}\|\phi-\psi\|_{C_{H}}^{2} \\
\quad+(L h+1) \int_{\tau}^{t}|w(s)|^{2} d s+|w(\tau)|^{2} \\
\quad+\alpha^{2}\|w(\tau)\|^{2} \\
\leq \int_{\tau}^{t}\|w\|^{2}\|u\| d s+L h^{2}\|\phi-\psi\|_{C_{H}}^{2} \\
\quad+(L h+1) \int_{\tau}^{t}|w(s)|^{2} d s+\|\phi-\psi\|_{C_{H}}^{2} \\
\quad+\alpha^{2}\|\phi-\psi\|_{C_{V}}^{2} \\
\leq C \int_{\tau}^{t}\|w(s)\|^{2} d s+C\|\phi-\psi\|_{C_{V}}^{2} .
\end{gathered}
$$

The last inequality comes from Poincaré inequality and the boundedness of $\|u\|$. Therefore, the Gronwall inequality implies the uniqueness of the solution. The proof is complete.

\section{Existence of Pullback Attractor}

In this section, we will prove the existence of pullback attractor to problem (10). First we give existence of pullback absorbing set for the process $\{U(t, \tau)\}$ generated by the global solution to problem (10).

Lemma 3. Assume $\left(H_{1}\right)-\left(H_{3}\right)$ hold and $L \leq v^{2} \lambda_{1}^{2} \varepsilon / 4 e^{\text {sh }}$; then, the process $\{U(t, \tau)\}$ is pullback dissipative, where $0<\varepsilon<$ $\min \left\{v / \alpha^{2}, v \lambda_{1} / 4\right\}$.

Proof. Multiplying (10) by $u$ and integrating over $\Omega$, we obtain

$$
\begin{aligned}
& \frac{\partial}{\partial t}\left(|u|^{2}+\alpha^{2}\|u\|^{2}\right)+2 v\|u\|^{2} \\
& \quad \leq \eta|u|^{2}+\frac{1}{\eta}\left|\int_{-h}^{0} G(s, u(t+s)) d s\right|^{2},
\end{aligned}
$$

where $\eta$ is a constant determined later.

By Poincaré inequality, we have

$$
\begin{aligned}
& \frac{\partial}{\partial t}\left(|u|^{2}+\alpha^{2}\|u\|^{2}\right)+\nu\|u\|^{2}+\left(\nu \lambda_{1}-\eta\right)|u|^{2} \\
& \quad \leq \frac{1}{\eta}\left|\int_{-h}^{0} G(s, u(t+s)) d s\right|^{2} .
\end{aligned}
$$

Since

$$
\begin{aligned}
e^{-\varepsilon t} & \frac{\partial}{\partial t}\left[e^{\varepsilon t}\left(|u|^{2}+\alpha^{2}\|u\|^{2}\right)\right] \\
& =\varepsilon\left(|u|^{2}+\alpha^{2}\|u\|^{2}\right)+\frac{\partial}{\partial t}\left(|u|^{2}+\alpha^{2}\|u\|^{2}\right),
\end{aligned}
$$

then

$$
\begin{aligned}
& e^{-\varepsilon t} \frac{\partial}{\partial t}\left[e^{\varepsilon t}\left(|u|^{2}+\alpha^{2}\|u\|^{2}\right)\right] \\
& \leq\left(\varepsilon+\eta-v \lambda_{1}\right)|u|^{2}+\left(\varepsilon \alpha^{2}-v\right)\|u\|^{2} \\
& \quad+\frac{1}{\eta}\left|\int_{-h}^{0} G(s, u(t+s)) d s\right|^{2} .
\end{aligned}
$$

Integrating (38) from $\tau$ to $t$, we get

$$
\begin{aligned}
& e^{\varepsilon t}\left(|u(t)|^{2}+\alpha^{2}\|u(t)\|^{2}\right) \\
& -e^{\varepsilon \tau}\left(|u(\tau)|^{2}+\alpha^{2}\|u(\tau)\|^{2}\right) \\
& \leq \int_{\tau}^{t} e^{\varepsilon s}\left[\left(\varepsilon+\eta-v \lambda_{1}\right)|u|^{2}+\left(\varepsilon \alpha^{2}-v\right)\|u\|^{2}\right] d s \\
& \quad+\frac{1}{\eta} \int_{\tau}^{t} e^{\varepsilon r}\left|\int_{-h}^{0} G(s, u(r+s)) d s\right|^{2} d r .
\end{aligned}
$$

Assumptions $\left(H_{1}\right)-\left(H_{3}\right)$ imply that

$$
\begin{aligned}
& \int_{\tau}^{t} e^{\varepsilon r}\left|\int_{-h}^{0} G(s, u(r+s)) d s\right|^{2} d r \\
& \leq \int_{\tau}^{t} e^{\varepsilon r} \int_{-h}^{0}|G(s, u(r+s))|^{2} d s d r \\
& \leq L \int_{-h}^{0} \int_{\tau}^{t} e^{\varepsilon r}|u(r+s)|^{2} d r d s \\
& \leq L \int_{-h}^{0} \int_{\tau+s}^{t+s} e^{\varepsilon(r-s)}|u(r)|^{2} d r d s \\
& \leq L \int_{-h}^{0} \int_{\tau-h}^{t} e^{\varepsilon(r-s)}|u(r)|^{2} d r d s \\
& \leq L \int_{-h}^{0} e^{-\varepsilon s} \int_{\tau-h}^{t} e^{\varepsilon r}|u(r)|^{2} d r d s \\
& \leq L \int_{-h}^{0} e^{-\varepsilon s}\left(\int_{\tau-h}^{\tau} e^{\varepsilon r}|u(r)|^{2} d r\right. \\
& \left.+\int_{\tau}^{t} e^{\varepsilon r}|u(r)|^{2} d r\right) d s \leq \frac{L e^{\varepsilon h}}{\varepsilon}\left(\frac{1}{\varepsilon}\|\phi\|_{C_{H}}^{2} e^{\varepsilon \tau}\right. \\
& \left.+\int_{\tau}^{t} e^{\varepsilon r}|u(r)|^{2} d r\right) \leq \frac{L e^{\varepsilon(\tau+h)}}{\varepsilon^{2}}\|\phi\|_{C_{H}}^{2}+\frac{L e^{\varepsilon h}}{\varepsilon} \\
& \cdot \int_{\tau}^{t} e^{\varepsilon r}|u(r)|^{2} d r .
\end{aligned}
$$

Substituting (40) into (39), we have

$$
\begin{aligned}
& e^{\varepsilon t}\left(|u(t)|^{2}+\alpha^{2}\|u(t)\|^{2}\right)-e^{\varepsilon \tau}\left(|u(\tau)|^{2}+\alpha^{2}\|u(\tau)\|^{2}\right) \\
& \leq \int_{\tau}^{t} e^{\varepsilon s}\left[\left(\varepsilon+\eta-v \lambda_{1}+\frac{L e^{\varepsilon h}}{2 \eta \varepsilon}\right)|u|^{2}\right. \\
& \left.+\left(\varepsilon \alpha^{2}-v\right)\|u\|^{2}\right] d s+\frac{L e^{\varepsilon(\tau+h)}}{2 \eta \varepsilon^{2}}\|\phi\|_{C_{H}}^{2} .
\end{aligned}
$$


Let $\eta=(1 / 2) \nu \lambda_{1}$, choosing $0<\varepsilon<\min \left\{\nu / \alpha^{2}, \nu \lambda_{1} / 4\right\} . L \leq$ $\nu^{2} \lambda_{1}^{2} \varepsilon / 4 e^{\varepsilon h}$ implies that

$$
\max \left\{\varepsilon+\eta-\nu \lambda_{1}+\frac{\eta^{-1} L e^{\varepsilon h}}{2 \varepsilon}, \varepsilon \alpha^{2}-\nu\right\}<0
$$

then,

$$
\begin{aligned}
& e^{\varepsilon t}\left(|u(t)|^{2}+\alpha^{2}\|u(t)\|^{2}\right) \\
& \quad \leq \frac{L e^{\varepsilon(\tau+h)}}{\varepsilon^{2} \nu \lambda_{1}}\|\phi\|_{C_{H}}^{2}+e^{\varepsilon \tau}\left(|u(\tau)|^{2}+\alpha^{2}\|u(\tau)\|^{2}\right),
\end{aligned}
$$

which implies

$$
\begin{aligned}
|u(t)|^{2}+\alpha^{2}\|u(t)\|^{2} & \\
\leq & \frac{L e^{\varepsilon(\tau+h-t)}}{\varepsilon^{2} \nu \lambda_{1}}\|\phi\|_{C_{H}}^{2} \\
& +e^{\varepsilon(\tau-t)}\left(|\phi(\tau)|^{2}+\alpha^{2}\|\phi(\tau)\|^{2}\right) .
\end{aligned}
$$

Now, if we take $t \geq \tau+h$, then, for $\theta \in[-h, 0]$, we have

$$
\begin{aligned}
|u(t+\theta)|^{2}+\alpha^{2}\|u(t+\theta)\|^{2} & \\
\leq & \frac{L e^{\varepsilon(\tau+h-t-\theta)}}{\varepsilon^{2} \nu \lambda_{1}}\|\phi\|_{C_{H}}^{2} \\
& +e^{\varepsilon(\tau-t-\theta)}\left(\|\phi\|_{C_{H}}^{2}+\alpha^{2}\|\phi\|_{C_{V}}^{2}\right) .
\end{aligned}
$$

We denote by $\mathscr{R}$ the set of all functions $r:(-\infty,+\infty) \rightarrow$ $(0,+\infty)$ such that

$$
\lim _{\tau \rightarrow-\infty} e^{\alpha \tau} r^{2}(\tau)=0
$$

Then, the closed ball in $C_{V}$ defined by

$$
B=\left\{\varphi \in C_{V}:\|\varphi\|_{C_{V}}^{2} \leq 1\right\}
$$

is pullback absorbing set for $\{U(t, \tau)\}$. The proof is complete.

We next prove the asymptotic compactness of solution to problem (10) by contractive functions; see $[19,20]$.

Let $X$ be a Banach space and let $B$ be a bounded subset of $X$. We call a function $\Phi(\cdot, \cdot)$ which, defined on $X \times X$, is a contractive function on $B \times B$ if for any sequence $\left\{x_{n}\right\}_{n=1}^{\infty} \subset B$ there is a subsequence $\left\{x_{n_{k}}\right\}_{k=1}^{\infty} \subset\left\{x_{n}\right\}_{n=1}^{\infty}$ such that

$$
\lim _{l \rightarrow \infty} \lim _{k \rightarrow \infty} \Phi\left(x_{n_{k}}, x_{n_{l}}\right)=0 .
$$

Denote all such contractive functions on $B \times B$ by $C(B)$.
Theorem 4 (see [19]). Let $\{S(t)\}_{t \geq \tau}$ be a semigroup on a Banach space $(X,\|\cdot\|)$ and have a bounded absorbing set $B$, $\forall \epsilon>0$; there exist $T=T(B, \epsilon)$ and $\Phi \in C(B)$, such that

$$
\|S(T) x-S(T) y\| \leq \epsilon+\Phi_{T}(x, y), \quad \forall x, y \in B
$$

where $\Phi_{T}$ depends on $T$. Then, $\{S(t)\}_{t \geq \tau}$ is asymptotically compact in $X$.

Lemma 5. Assume that $\left(H_{1}\right)-\left(H_{3}\right)$ hold; the process $\{U(t, \tau)\}_{t \geq \tau}$ generated by the global solution to problem (10) is asymptotically compact.

Proof. Let $u^{i}(t)$ be the solution to problem (10) with initial data $\phi^{i} \in B(i=1,2)$, respectively. Denote $v(t)=u^{1}(t)-u^{2}(t)$; then, $v(t)$ satisfies the equivalent abstract equation

$$
\begin{gathered}
\frac{\partial}{\partial t}\left(v+\alpha^{2} A v\right)+v A v+B\left(u^{1}, u^{1}\right)-B\left(u^{2}, u^{2}\right) \\
\quad=\int_{-h}^{0}\left[G\left(s, u^{1}(t+s)\right)-G\left(s, u^{2}(t+s)\right)\right] d s
\end{gathered}
$$

with the initial condition $v(t)=\phi^{1}(t-\tau)-\phi^{2}(t-\tau), t \in$ $[\tau-h, \tau]$.

Set an energy function

$$
E_{v}(t)=\frac{1}{2} \int_{\Omega}|v|^{2} d x+\frac{\alpha^{2}}{2} \int_{\Omega}|\nabla v|^{2} d x .
$$

Multiplying (50) by $v$ and integrating over $[s, T] \times \Omega$ with $T>$ $t+\tau, s \geq \tau$, we have

$$
\begin{aligned}
E_{v}(T) & -E_{v}(s)+v \int_{s}^{T} \int_{\Omega}|\nabla v|^{2} d x d r \\
& +\int_{s}^{T} \int_{\Omega}\left[B\left(u^{1}\right)-B\left(u^{2}\right)\right] v(r) d x d r \\
= & \int_{s}^{T} \int_{\Omega} \int_{-h}^{0}\left[G\left(\sigma, u^{1}(r+\sigma)\right)-G\left(\sigma, u^{2}(r+\sigma)\right)\right] v(r) d \sigma d x d r
\end{aligned}
$$

then we have

$$
\begin{aligned}
& v \int_{s}^{T} \int_{\Omega}|\nabla v|^{2} d x d r \leq E_{v}(s)-\int_{s}^{T} \int_{\Omega}\left[B\left(u^{1}\right)-B\left(u^{2}\right)\right] v(r) d x d r \\
& \quad+\int_{s}^{T} \int_{\Omega} \int_{-h}^{0}\left[G\left(\sigma, u^{1}(r+\sigma)\right)-G\left(\sigma, u^{2}(r+\sigma)\right)\right] v(r) d \sigma d x d r .
\end{aligned}
$$


Using Poincaré inequality and (51) and (53), we have

$$
\begin{aligned}
\int_{\tau}^{T} E_{v}(s) d s= & \frac{1}{2} \int_{\tau}^{T} \int_{\Omega}|v|^{2} d x d r+\frac{\alpha^{2}}{2} \int_{\tau}^{T} \int_{\Omega}|\nabla v|^{2} d x d r \leq C \int_{\tau}^{T} \int_{\Omega}|\nabla v|^{2} d x d r \\
\leq & C E_{v}(\tau)-C \int_{\tau}^{T} \int_{\Omega}\left[B\left(u^{1}\right)-B\left(u^{2}\right)\right] v(r) d x d r \\
& +C \int_{\tau}^{T} \int_{\Omega} \int_{-h}^{0}\left[G\left(\sigma, u^{1}(r+\sigma)\right)-G\left(\sigma, u^{2}(r+\sigma)\right)\right] v(r) d \sigma d x d r .
\end{aligned}
$$

Integrating (52) from $\tau$ to $T$ with respect to $s$, we obtain

$$
\begin{aligned}
T E_{v}(T)+v \int_{\tau}^{T} \int_{s}^{T} \int_{\Omega}|\nabla v|^{2} d x d r d s= & \int_{\tau}^{T} E_{v}(s) d s-\int_{\tau}^{T} \int_{s}^{T} \int_{\Omega}\left[B\left(u^{1}\right)-B\left(u^{2}\right)\right] v(r) d x d r d s \\
& +\int_{\tau}^{T} \int_{s}^{T} \int_{\Omega} \int_{-h}^{0}\left[G\left(\sigma, u^{1}(r+\sigma)\right)-G\left(\sigma, u^{2}(r+\sigma)\right)\right] v(r) d \sigma d x d r d s .
\end{aligned}
$$

Substituting (54) into (55), we get

$$
\begin{aligned}
T E_{v}(T)+v \int_{\tau}^{T} \int_{s}^{T} \int_{\Omega}|\nabla v|^{2} d x d r d s \leq & C E_{v}(\tau)-C \int_{\tau}^{T} \int_{\Omega}\left[B\left(u^{1}\right)-B\left(u^{2}\right)\right] v(r) d x d r \\
& +C \int_{\tau}^{T} \int_{\Omega} \int_{-h}^{0}\left[G\left(\sigma, u^{1}(r+\sigma)\right)-G\left(\sigma, u^{2}(r+\sigma)\right)\right] v(r) d \sigma d x d r \\
& -\int_{\tau}^{T} \int_{s}^{T} \int_{\Omega}\left[B\left(u^{1}\right)-B\left(u^{2}\right)\right] v(r) d x d r d s \\
& +\int_{\tau}^{T} \int_{s}^{T} \int_{\Omega} \int_{-h}^{0}\left[G\left(\sigma, u^{1}(r+\sigma)\right)-G\left(\sigma, u^{2}(r+\sigma)\right)\right] v(r) d \sigma d x d r d s
\end{aligned}
$$

Set

$$
\begin{aligned}
C_{0}= & C E_{v}(\tau)=\frac{C}{2} \int_{\Omega}\left|\phi^{1}(0)-\phi^{2}(0)\right|^{2} d x+\frac{C \alpha^{2}}{2} \int_{\Omega}\left|\nabla \phi^{1}(0)-\nabla \phi^{2}(0)\right|^{2} d x \\
\Phi\left(u^{1}, u^{2}\right)= & -C \int_{\tau}^{T} \int_{\Omega}\left[B\left(u^{1}\right)-B\left(u^{2}\right)\right] v(r) d x d r \\
& +C \int_{\tau}^{T} \int_{\Omega} \int_{-h}^{0}\left[G\left(\sigma, u^{1}(r+\sigma)\right)-G\left(\sigma, u^{2}(r+\sigma)\right)\right] v(r) d \sigma d x d r \\
& -\int_{\tau}^{T} \int_{s}^{T} \int_{\Omega}\left[B\left(u^{1}\right)-B\left(u^{2}\right)\right] v(r) d x d r d s \\
& +\int_{\tau}^{T} \int_{s}^{T} \int_{\Omega} \int_{-h}^{0}\left[G\left(\sigma, u^{1}(r+\sigma)\right)-G\left(\sigma, u^{2}(r+\sigma)\right)\right] v(r) d \sigma d x d r d s
\end{aligned}
$$


then, by (56), we have

$$
E_{v}(T) \leq \frac{C_{0}}{T}+\frac{1}{T} \Phi\left(u^{1}, u^{2}\right)
$$

a contractive function, since $B$ is a bounded positive invariant set.

If $u^{m} \rightarrow u(m \rightarrow \infty)$, we have the limits

One has $\forall \epsilon>0$; we choose $T>0$ large enough such that $C_{0} / T<\epsilon$. By Theorem 4 , it suffices to prove that $\Phi\left(u^{1}, u^{2}\right)$ is

$$
\begin{aligned}
& \lim _{n \rightarrow \infty} \lim _{m \rightarrow \infty} \int_{\tau}^{T} \int_{\Omega}\left(B\left(u^{n}\right)-B\left(u^{m}\right)\right)\left(u^{n}-u^{m}\right) d x d r \\
& =\lim _{n \rightarrow \infty} \lim _{m \rightarrow \infty} \int_{\tau}^{T} \int_{\Omega}\left[\left(\left(u^{n}-u^{m}\right) \cdot \nabla\right) u^{n}-\left(u^{m} \cdot \nabla\right)\left(u^{n}-u^{m}\right)\right]\left(u^{n}-u^{m}\right) d x d r=0, \\
& \lim _{n \rightarrow \infty} \lim _{m \rightarrow \infty} \int_{\tau}^{T} \int_{s}^{T} \int_{\Omega}\left(B\left(u^{n}\right)-B\left(u^{m}\right)\right)\left(u^{n}-u^{m}\right) d x d r d s \\
& =\lim _{n \rightarrow \infty} \lim _{m \rightarrow \infty} \int_{\tau}^{T} \int_{s}^{T} \int_{\Omega}\left[\left(\left(u^{n}-u^{m}\right) \cdot \nabla\right) u^{n}-\left(u^{m} \cdot \nabla\right)\left(u^{n}-u^{m}\right)\right]\left(u^{n}-u^{m}\right) d x d r d s=0 .
\end{aligned}
$$

By $\left(H_{1}\right)-\left(H_{3}\right)$, we have

$$
\begin{aligned}
& \lim _{n \rightarrow \infty} \lim _{m \rightarrow \infty} \int_{\tau}^{T} \int_{\Omega} \int_{-h}^{0}\left(G\left(\sigma, u^{n}(r+\sigma)\right)-G\left(\sigma, u^{m}(r+\sigma)\right)\right) v(r) d \sigma d x d r \\
& \leq \lim _{n \rightarrow \infty} \lim _{m \rightarrow \infty} \int_{\tau}^{T} \int_{\Omega} \int_{-h}^{0} L\left|u^{n}(r+\sigma)-u^{m}(r+\sigma)\right|\left|u^{n}(r)-u^{m}(r)\right| d \sigma d x d r=0, \\
& \lim _{n \rightarrow \infty} \lim _{m \rightarrow \infty} \int_{\tau}^{T} \int_{s}^{T} \int_{\Omega} \int_{-h}^{0}\left(G\left(\sigma, u^{n}(r+\sigma)\right)-G\left(\sigma, u^{m}(r+\sigma)\right)\right) v(r) d \sigma d x d r d s \\
& \leq \lim _{n \rightarrow \infty} \lim _{m \rightarrow \infty} \int_{\tau}^{T} \int_{s}^{T} \int_{\Omega} \int_{-h}^{0} L\left|\left(u^{n}-u^{m}\right)(r+\sigma)\right|\left|\left(u^{n}-u^{m}\right)(r)\right| d \sigma d x d r d s=0 .
\end{aligned}
$$

Combining (60)-(61) with (58), we have that $\Phi\left(u^{1}, u^{2}\right)$ is a contractive function.

The proof is complete.

Theorem 6. Assume that $\left(H_{1}\right)-\left(H_{3}\right)$ hold and $L \leq v^{2} \lambda_{1}^{2} \varepsilon /$ $4 e^{\varepsilon h}$; then, the process $\{U(t, \tau)\}$ generated by the global solution for problem (10) has a pullback attractor.

Proof. Lemma 3 implies $\{U(t, \tau)\}$ has a pullback absorbing set $B$ and Lemma 5 implies $\{U(t, \tau)\}$ is asymptotically compact; we obtain the conclusion immediately.

According to Theorem $6,0<\varepsilon<\min \left\{\nu / \alpha^{2}, \nu \lambda_{1} / 4\right\}$ and $L \leq v^{2} \lambda_{1}^{2} \varepsilon / 4 e^{\varepsilon h}$. Under the assumptions $\left(H_{1}\right)-\left(H_{3}\right)$, the NSV equation (1) with a distributed delay has a pullback attractor.

\section{Conflict of Interests}

The authors declare that they have no competing interests.

\section{Authors' Contribution}

Yantao Guo carried out the long time behavior of solutions. Shuilin Cheng carried out the pullback attractor. Yanbin Tang carried out the distributed delay. All authors read and approved the final paper.

\section{Acknowledgment}

This work was supported by the National Natural Science Foundation of China (Grant nos. 11471129 and 11272277).

\section{References}

[1] A. P. Oskolkov, "The uniqueness and solvability in the large of boundary value problems for the equations of motion of aqueous solutions of polymers," Zapiski Nauchnykh Seminarov Leningrad Otdel Mathematics Institute Stekov (LOMI), vol. 38, pp. 98-136, 1973. 
[2] Y. Cao, E. M. Lunasin, and E. S. Titi, "Global well-posedness of the three-dimensional viscous and inviscid simplified Bardina turbulence models," Communications in Mathematical Sciences, vol. 4, no. 4, pp. 823-848, 2006.

[3] V. K. Kalantarov and E. S. Titi, "Global attractors and determining modes for the 3D Navier-Stokes-Voight equations," Chinese Annals of Mathematics, Series B, vol. 30, no. 6, pp. 697-714, 2009.

[4] G.-C. Yue and C.-K. Zhong, "Attractors for autonomous and nonautonomous 3D Navier-Stokes-Voight equations," Discrete and Continuous Dynamical Systems Series B, vol. 16, no. 3, pp. 985-1002, 2011.

[5] J. García-Luengo, P. Marín-Rubio, and J. Real, "Pullback attractors for three-dimensional non-autonomous Navier-StokesVoigt equations," Nonlinearity, vol. 25, no. 4, pp. 905-930, 2012.

[6] J. Hale, Theory of Functional Differential Equations, Springer, New York, NY, USA, 1977.

[7] Y. Tang and M. Wang, "A remark on exponential stability of time-delayed Burgers equation," Discrete and Continuous Dynamical Systems. Series B, vol. 12, no. 1, pp. 219-225, 2009.

[8] Y. Tang and L. Zhou, "Stability switch and Hopf bifurcation for a diffusive prey-predator system with delay," Journal of Mathematical Analysis and Applications, vol. 334, no. 2, pp. 1290-1307, 2007.

[9] J. H. Wu, Theory and Applications of Partial Differential Equations, Springer, New York, NY, USA, 1996.

[10] L. Zhou, Y. B. Tang, and S. Hussein, "Stability and Hopf bifurcation for a delay competition diffusion system," Chaos, Solitons \& Fractals, vol. 14, no. 8, pp. 1201-1225, 2002.

[11] T. Caraballo and J. Real, "Navier-Stokes equations with delays," The Royal Society of London. Proceedings. Series A. Mathematical, Physical and Engineering Sciences, vol. 457, no. 2014, pp. 2441-2453, 2001.

[12] T. Caraballo and J. Real, "Asymptotic behaviour of two-dimensional Navier-Stokes equations with delays," The Royal Society of London. Proceedings. Series A. Mathematical, Physical and Engineering Sciences, vol. 459, no. 2040, pp. 3181-3194, 2003.

[13] T. Caraballo and J. Real, "Attractors for 2D-Navier-Stokes models with delays," Journal of Differential Equations, vol. 205, no. 2, pp. 271-297, 2004.

[14] Z. Hu and Y. Wang, "Pullback attractors for a nonautonomous nonclassical diffusion equation with variable delay," Journal of Mathematical Physics, vol. 53, no. 7, Article ID 072702, 17 pages, 2012.

[15] H. Y. Li and Y. M. Qin, "Pullback attractors for three-dimensional navier-stokes-voigt equations with delays," Boundary Value Problems, vol. 2013, article 191, 2013.

[16] P. Marín-Rubio and J. Real, "Attractors for 2D-Navier-Stokes equations with delays on some unbounded domains," Nonlinear Analysis. Theory, Methods \& Applications, vol. 67, no. 10, pp. 2784-2799, 2007.

[17] P. Marín-Rubio, J. Real, and J. Valero, "Pullback attractors for a two-dimensional Navier-Stokes model in an infinite delay case," Nonlinear Analysis: Theory, Methods \& Applications, vol. 74, no. 5, pp. 2012-2030, 2011.

[18] P. Marín-Rubio and J. Real, "Pullback attractors for 2D-NavierStokes equations with delays in continuous and sub-linear operators," Discrete and Continuous Dynamical Systems. Series A, vol. 26, no. 3, pp. 989-1006, 2010.
[19] A. K. Khanmamedov, "Global attractors for wave equations with nonlinear interior damping and critical exponents," Journal of Differential Equations, vol. 230, no. 2, pp. 702-719, 2006.

[20] L. Yang and C.-K. Zhong, "Global attractor for plate equation with nonlinear damping," Nonlinear Analysis: Theory, Methods \& Applications, vol. 69, no. 11, pp. 3802-3810, 2008. 


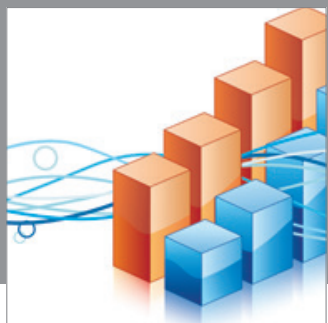

Advances in

Operations Research

mansans

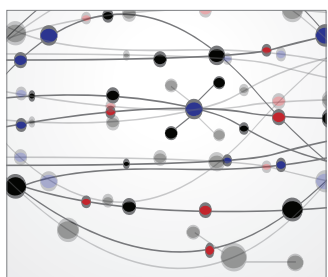

The Scientific World Journal
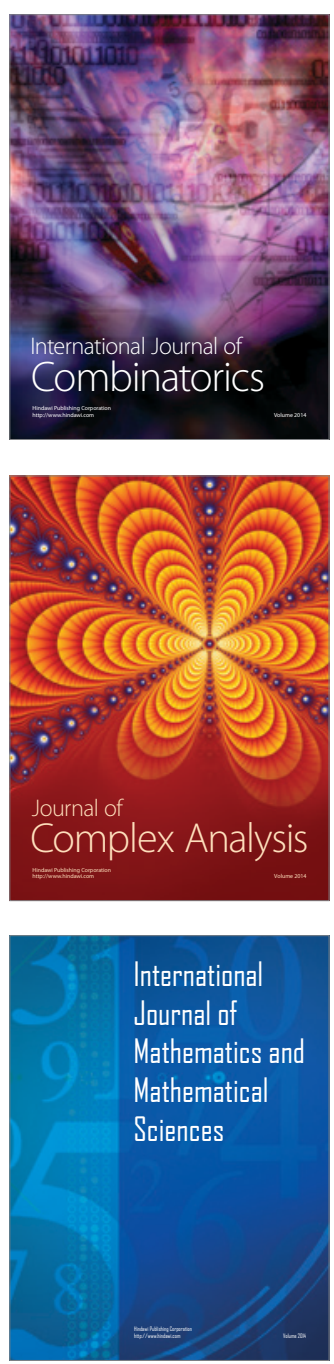
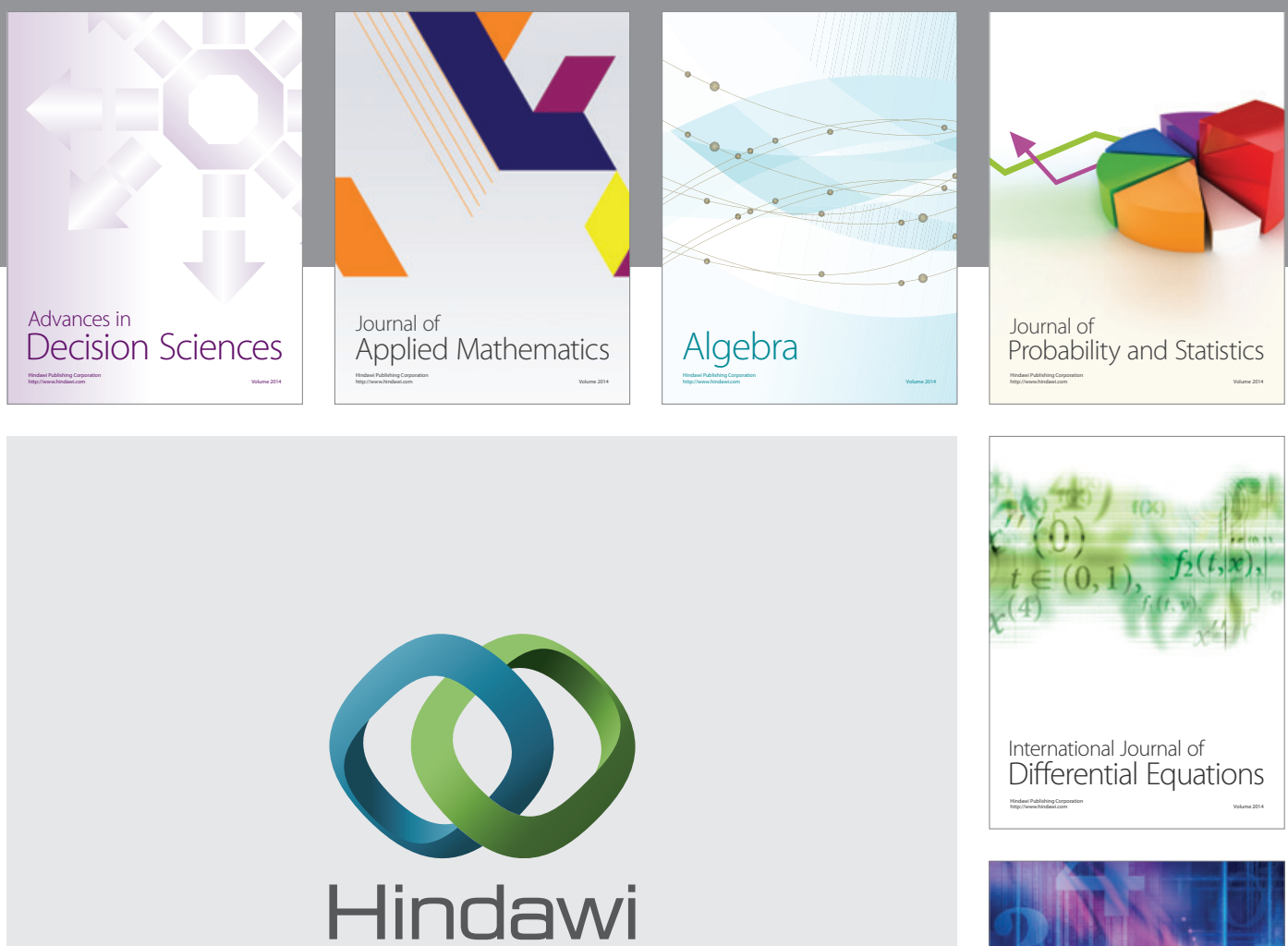

Submit your manuscripts at http://www.hindawi.com
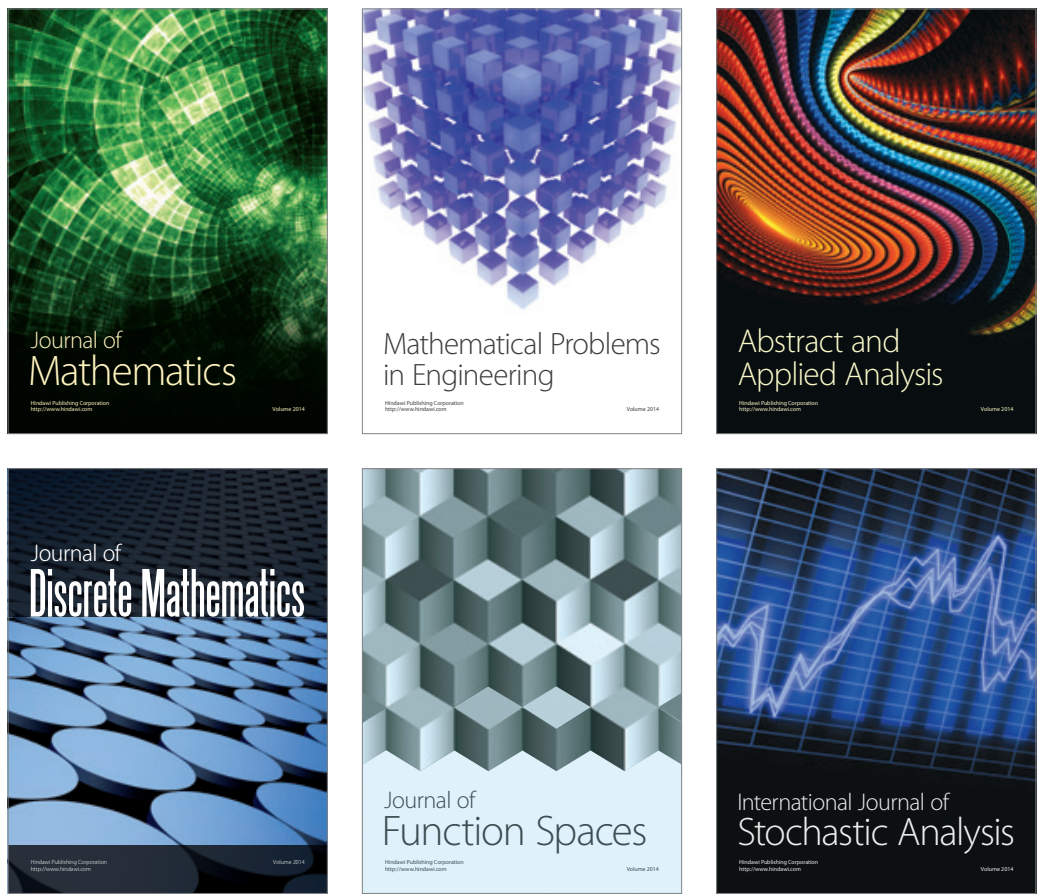

Journal of

Function Spaces

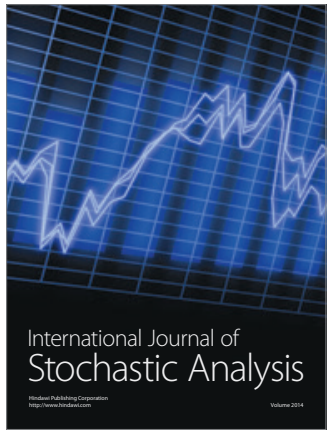

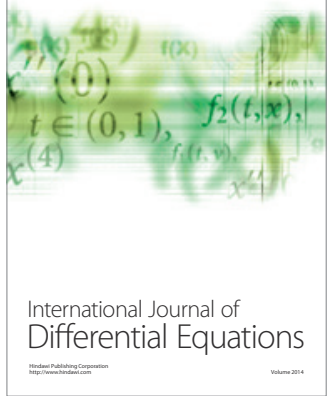
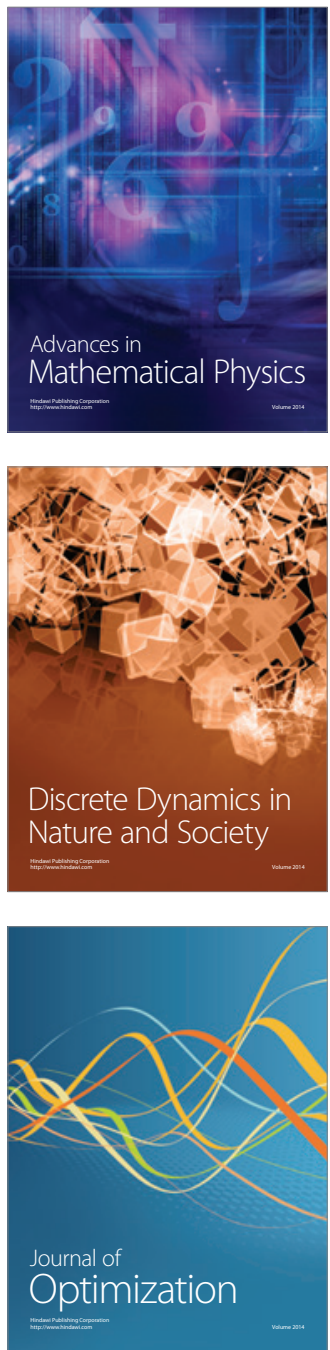